\title{
Mechanical Impedance Matching Using a Magnetic Linear Gear
}

\author{
Ignacio Valiente-Blanco, ${ }^{1}$ Cristian Cristache, ${ }^{1}$ Juan Sanchez-Garcia-Casarrubios, ${ }^{1}$ \\ Fernando Rodriguez-Celis, ${ }^{1}$ and Jose-Luis Perez-Diaz ${ }^{2}$
}

\author{
${ }^{1}$ MAG SOAR SL, 28341 Valdemoro, Spain \\ ${ }^{2}$ Teoría de la Señal y Comunicaciones, Escuela Politécnica, Universidad de Alcalá, Campus Universitario, \\ Ctra. Madrid-Barcelona, Km 33,6, Madrid, Spain
}

Correspondence should be addressed to Ignacio Valiente-Blanco; ivaliente@magsoar.com

Received 27 March 2017; Revised 13 June 2017; Accepted 2 July 2017; Published 21 August 2017

Academic Editor: Onome E. Scott-Emuakpor

Copyright (c) 2017 Ignacio Valiente-Blanco et al. This is an open access article distributed under the Creative Commons Attribution License, which permits unrestricted use, distribution, and reproduction in any medium, provided the original work is properly cited.

\begin{abstract}
As part of the Fp7 Clean Sky Project, a linear magnetic gear prototype, called Z-transmitter, for aerospace application was designed, built, and tested. It demonstrates a maximum force capacity of $4700 \mathrm{~N}$ at $25^{\circ} \mathrm{C}$ and $4500 \mathrm{~N}$ at $90^{\circ} \mathrm{C}$. Force ratio between slow and fast stages remains constant and equal to the design value: 7.0. The behavior of the real $Z$-transmitter as a mechanical impedance matching device when any stiffness is attached to the fast stage including the limit cases of a blocked fast stage or a free to move fast stage is experimentally explored. Although the real $Z$-transmitter deviates from the ideal, frictionless and massless, device, it still provides an impedance matching effect large enough to potentially become an extremely useful technology for vibration control when combined with other elements such as dampers, springs, or active elements.
\end{abstract}

\section{Introduction}

Open rotor engines present a huge potential for reductions in fuel consumption and pollution relative to turbofan engines of equivalent thrust. The development and use of these engines mounted on the fuselage are indeed conditioned by the availability of a technology for effectively reducing the transmission of vibrations to the aircraft structure.

However, the frontiers for vibration isolation engineering are currently hard to expand. The biggest challenge is to find a vibration technology capable of providing an optimum performance at both low and high frequency ranges without significantly increasing the cost or weight of the system ([1] and references therein).

Well known classical vibration isolation is based on the use of very low stiffness combined with large masses to reduce the resonant frequency of the system [2]. Passive vibration isolation systems are typically used in a great range of applications to provide high performance and stability without the need for external power. Some common passive solutions are viscous fluid dampers or viscoelastic composite dampers. Magnetic damping is also a passive damping solution based on induction of electrical eddy currents [3].
These technologies are especially interesting because of their contactless working principle. The absence of friction eliminates lubricant requirements and increases the system lifetime. Recent and innovative configurations are currently developing the damping capacity of this technology $[4,5]$. However, magnetic damping shares the common limitation of pure passive vibration isolation techniques: the incapability of damping low frequency microvibrations.

Active isolation methods have been proposed as a tunable option to deal with low frequency vibrations [6]; however, external energy is required for driving the additional actuators and sensors, resulting in an increase in the overall cost, weight, and stability.

Other options to battle low frequency vibrations are active/passive hybrid isolation, semiactive systems, and smart passive combinations of positive and negative stiffness [7]. However, up to now, optimal damping of both low and high frequencies has not been accomplished and the idoneal solution depends on the concrete application.

A possible solution is to multiply low frequency vibrations in order to convert them into easier to damp high frequency vibrations. Yilmaz and Kikuchi [8] proposed a filter-like vibration isolator using a simple lever mechanism 
to multiply the effects of a coupled inertia. However, simple lever mechanisms are not quite practical for their size and hysteresis of bearings. Other motion multiplier mechanisms like conventional gear cannot be used due to the large hysteretic forces. This has prevented the existence of efficient mechanical impedance transformers or matching elements suitable for enhancing vibration isolation.

The Fp7 Clean Sky Project Z-damper had the objective of developing a new technology for the reduction of vibrations suitable for such a tough environment. Moreover, the ambitious objectives of this project included achieving a real breakthrough in vibration control technologies creating an absolutely new kind of mechanical impedance matching device as a key tool for enhancing the effectiveness of other vibration control elements.

In order to achieve these objectives a device based on noncontact linear magnetic gear was designed. Some of us [10] suggested that linear magnetic gears can provide mechanical impedance matching of a vibration control device, that is, a damper, an active actuator, or a tuned vibration absorber, and be used to multiply its effect in the same way that electrical transformers are used in electrical engineering.

The basic idea is simple: the gear multiplies the movement of the vibrating element to be damped driving a fast stage that can be more easily damped by a damper or driven by an active control.

This paper represents a major step forward since the theoretical model introduced in the previous publication [9]. The present work exposes the first experimental results evidencing the validity of the preliminary idea.

\section{Physics of the $Z$-Transmitter}

Linear magnetic gears are devices able to amplify the displacement of any oscillating mechanical element [10]. As they are the linear version of rotational magnetic gear [11] they are also expected to inherit their interesting properties like not needing lubrication, zero backlash, large temperature range, and long working life [12]. Although some screw-rotational magnetic gear has been proposed for vibration damping [13] a pure linear geometry is much simpler to manufacture.

A linear magnetic gear is provided with a wave generator (typically a fast-moving stage) which generates a magnetic wave that interacts with an outer part composed of soft magnetic materials and a linear permanent magnet spline. Figure 1 shows a schematic representation of the three main parts of the device: slow stage, stator, and fast stage. The interaction between a set of ring magnets and soft magnetic parts provided a multiplication of the movement according to a precise algebra as described elsewhere [14]: in an elementary gear unit the number of magnetic teeth in the outer soft magnetic part $n_{0}$ and the number of magnetic teeth in the linear spline $n_{s}$ will differ in just 1 and the gear ratio will be $1: n_{0}$.

In such a linear gear mechanism, provided there is no friction or dissipative effect and according to the principle of virtual works, the force is divided by $n$. The stator exerts a

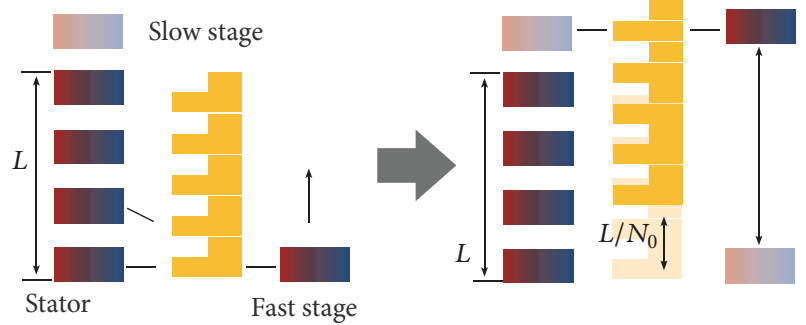

FIGURE 1: Magnetic linear impedance coupler concept.

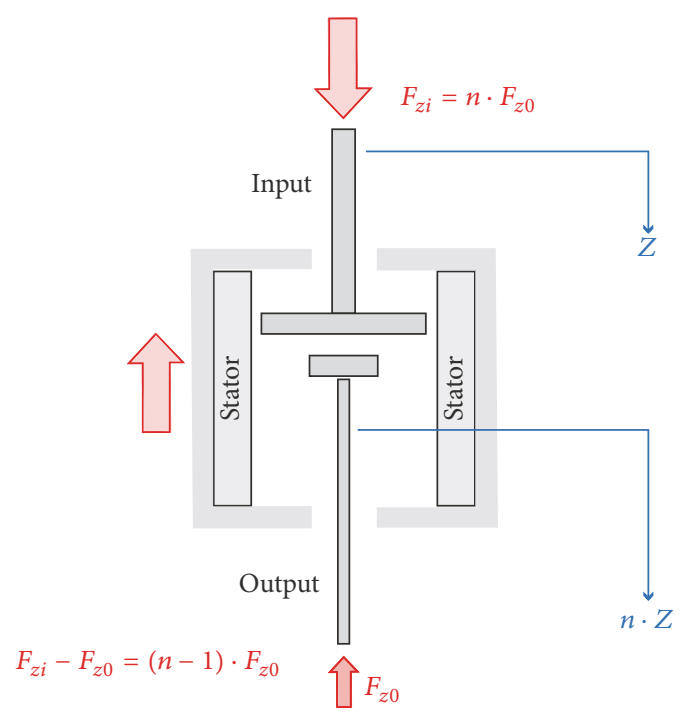

FIGURE 2: Scheme of the sum of forces applied by $Z$-transmitter.

force into the ground such that the total sum of forces applied by the system is zero as shown in Figure 2.

If the device has any internal friction, for example, that of bearings, then the output force will be decreased with a magnitude equal to the friction. For an ideal linear gear with zero friction whose internal elements were massless then the input/output impedance ratio could be written as

$$
n=\frac{F_{\text {input }}}{F_{\text {output }}}=\frac{X_{\text {output }}}{X_{\text {input }}} .
$$

A simple example of how this can be used to match impedances is the configuration of Figure 3 in which a spring with stiffness $K$ is matched to a suspended mass $M$ through a $Z$-transmitter with ratio $n$. The mass is connected to the ground through a damper with coefficient $C$ as well.

When an external oscillating force $F_{e}$ is applied, we can write the following equations of motion:

$$
\begin{aligned}
m \cdot \ddot{Z}+C \dot{Z} & =F_{e}-F_{z i}, \\
F_{z i} & =n \cdot F_{z o}=n^{2} \cdot K Z, \\
m \cdot \ddot{Z}+C \dot{Z}+n^{2} \cdot K Z & =F_{e} .
\end{aligned}
$$

While the force transmitted to the ground is

$$
F_{\equiv}=C \dot{Z}+(n-1) F_{z 0}+F_{z 0}=C \dot{Z}+n^{2} \cdot K Z,
$$




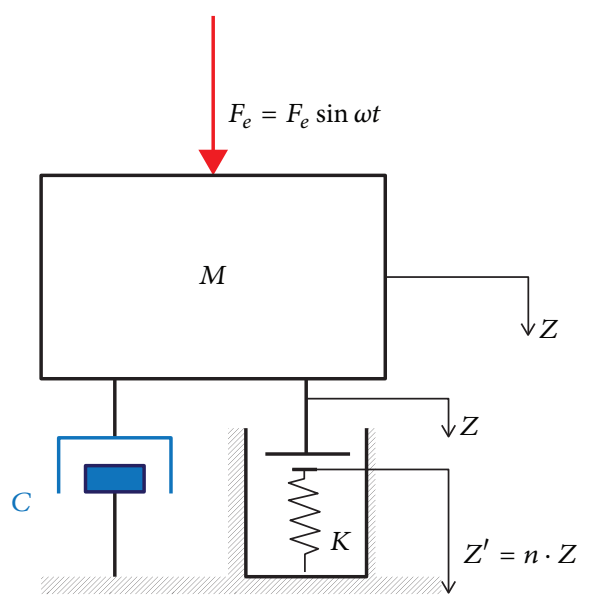

FIGURE 3: Impedance matching of a spring to a suspended mass trough a $Z$-transmitter.

therefore the transmissibility reads

$$
T=\left|\frac{F_{\equiv}}{F_{e}}\right|=\sqrt{\frac{\left(n^{2} \cdot K\right)^{2}+(C \omega)^{2}}{\left(n^{2} K-m \omega^{2}\right)^{2}+(C \omega)^{2}}} .
$$

Or in terms of the nondimensionalized frequency $\Omega=$ $\omega / \sqrt{K / M}$,

$$
T=\left|\frac{F_{\equiv}}{F_{e}}\right|=\sqrt{\frac{\left(n^{4}+(2 c)^{2}\right)}{\left(n^{2}-\Omega^{2}\right)^{2}+(2 \xi \Omega)^{2}}} .
$$

With phase:

$$
\theta_{T C}=\operatorname{arctg}\left(\frac{2 \xi \Omega^{3}}{\left(n^{4}+\left(4 \xi^{2}-n^{2}\right) \cdot \Omega^{2}\right)}\right) .
$$

The critical damping coefficient $C_{c}$ is found to be

$$
m \cdot \ddot{Z}+C \dot{Z}=F_{e}-F_{z i} \cdot
$$

This means that critical damping will occur at values $n$ times larger than that of the case in which no $Z$-transmitter is used (or $n=1$ ). It is to be noted that $n$ can be designed to be both greater and smaller than 1 .

Figure 4 shows the transmissibility for a value of the damping coefficient $\xi=0.1$ and a set of values of the gear ratio $n$. How the gear ratio $n$ affects both the resonance frequency and the damping characteristic (becoming supercritical for $n$ lower than 1) is clear.

Figure 5 reflects the corresponding relation between the transmissibility phase and the gear ratio $n$.

\section{Prototype}

Particularly, the $Z$-transmitter was designed as a linear magnetic gear with a 1:7 ratio in a coaxial configuration. In the present case, any displacement of the slow stage makes the

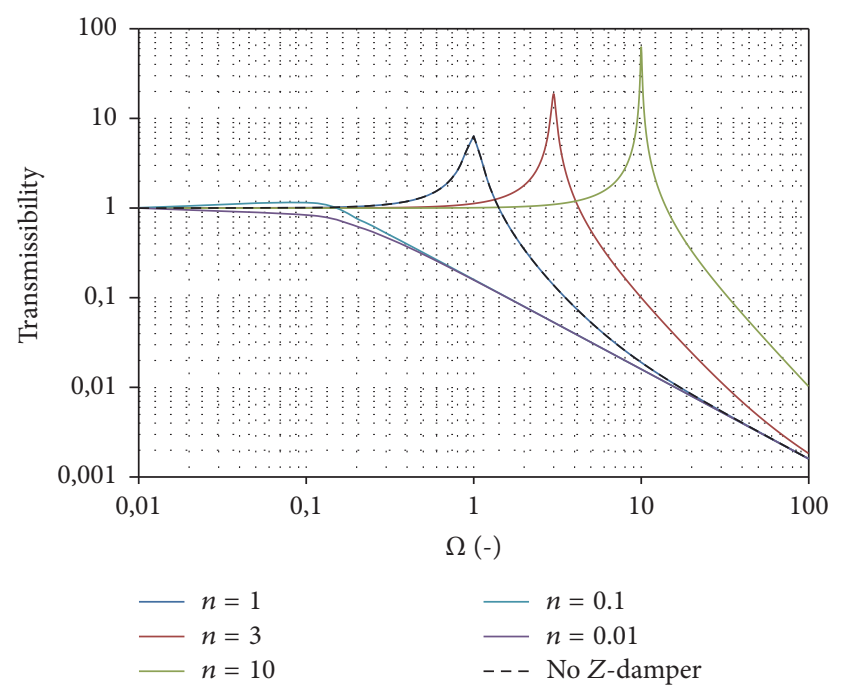

Figure 4: Transmissibility module versus frequency ratio for the $Z$ damper stiffness configuration. Damping ratio $\xi=0.1$.

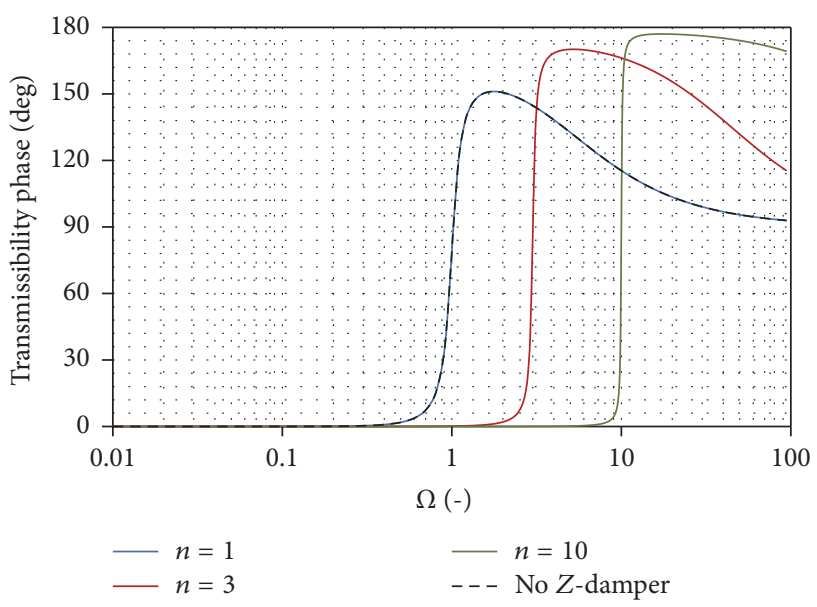

FIgURE 5: Transmissibility phase versus frequency ratio for the $Z$ damper stiffness configuration. Damping ratio $\xi=0.1$.

fast stage move seven times longer. A key expected property of magnetic gears, unlike mechanical gears, is that this multiplication must be sensitive to any small displacement without any backlash.

The prototype weighed $9 \mathrm{~kg}$ and was $490 \mathrm{~mm}$ long and with a diameter of $80 \mathrm{~mm}$. It was designed to provide up to $5 \mathrm{kN}$. The maximum amplitude acceptable for the slow stage is $5 \mathrm{~mm}$ ( $10 \mathrm{~mm}$ stroke) corresponding to a $35 \mathrm{~mm}$ amplitude for the fast stage. There are some ends to prevent the movement beyond the range limits. It has been provided with friction linear bearings enabling high-temperature working range in spite of their poorer performance compared to ball bearings.

The effect of the friction of the bearings and masses of the elements as well as any dissipative magnetic interaction cannot be easily modelled and should be determined experimentally. Figure 6 shows the once built $Z$-transmitter prototype, ready for being tested. 


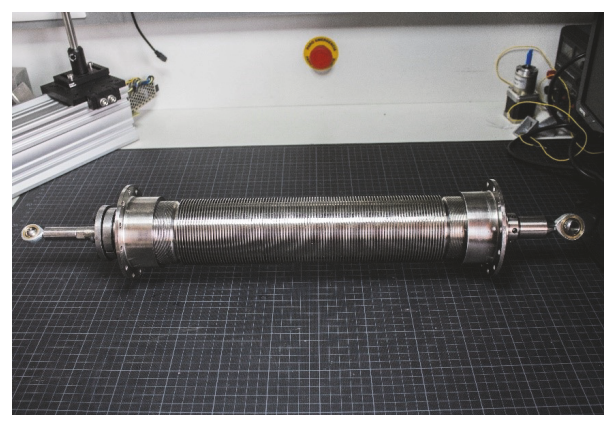

FIGURE 6: $Z$-transmitter prototype.

\section{Experimental Setup and Procedure}

A test bench was specifically designed and constructed. It was provided with a hydraulic actuator to generate a vibration with a force up to $15 \mathrm{kN}$ and amplitude up to $15 \mathrm{~mm}$-in a bandwidth from $0.01 \mathrm{~Hz}$ to $60 \mathrm{~Hz}$ at a temperature ranging from $-50^{\circ} \mathrm{C}$ to $250^{\circ} \mathrm{C}$. It is provided with load cells to measure the force exerted on both stages as well as a LVDT position sensor in the slow stage. Figure 7 shows a scheme of the main components and Figure 8 shows a picture of the test bench installation. A closed air flow is controlled to provide controlled temperature and speed.

Three setups were used to characterize the properties of the prototype. In a first setup, both the stator and fast stage of the $Z$-transmitter are tightly fixed to the bench while the slow stage can be displaced quasistatically. As the fast stage is blocked the force exerted by the actuator quickly increases. If it surpasses a certain threshold (maximum admissible force) then the slow stage slides, as previously described in magnetic gears [15]. We define the equivalent stiffness of slow and fast stages as follows:

$$
\begin{aligned}
& K(x)_{\text {slow }}=\frac{d F_{\text {slow }}(x)}{d X_{\text {slow }}(x)}, \\
& K(x)_{\text {fast }}=\frac{d F_{\text {fast }}(x)}{d X_{\text {fast }}(x)} .
\end{aligned}
$$

Taking into account (1) we can write

$$
n=\sqrt{\frac{K(x)_{\text {slow }}}{K(x)_{\text {fast }}}}
$$

In a perfect theoretical device, this ratio should equal the geometric speed ratio that was designed to be 7 in the prototype.

A second setup will be obtained by releasing the fast stage to move freely applying a vibration movement to the slow stage at a defined frequency from 0 up to $50 \mathrm{~Hz}$. The position of the fast stage is measured using a laser sensor. With no external load, the fast stage should ideally follow the movement of the slow stage multiplied by 7. A real $Z$ transmitter prototype will be strongly affected by inertia and magnet interaction stiffness.

Last, a third setup will be like the second one but connecting a traction-compression spring attached to the fast
TABLE 1: $Z$-transmitter slow and fast stages stiffness at $15^{\circ} \mathrm{C}$.

\begin{tabular}{lc}
\hline$K_{S}$ slow equivalent stiffness at equilibrium & $2200 \pm 140 \mathrm{~N} / \mathrm{mm}$ \\
$K_{F}$ fast equivalent stiffness at equilibrium & $48 \pm 8 \mathrm{~N} / \mathrm{mm}$ \\
$n_{e}$ experimental force ratio at equilibrium & $6.7 \pm 0.8$ \\
\hline
\end{tabular}

stage and to the bench structure. Both, the stator of the device and the attached springs are connected to the bench structure (considered as the ground of the system). Figure 9 shows a schematic representation of this setup and Figure 10 shows a picture of the spring eventually used for this setup. This setup is intended to test the multiplier capacity of the device when attached to the linear impedance of a spring.

Two springs were coupled together and installed in series with the fast stage of the $Z$-transmitter as shown in Figure 10. The geometry of the system and a preload ensures that at least one of the springs is always under compression and prevents backlash in the central position. Additionally, the symmetry of the setup ensures that compression and traction stiffness are equal. The total mass of the fast stage of the $Z$-transmitter plus attached elements (shafts, joints, etc.) was measured to be $5.0 \pm 0.1 \mathrm{~kg}$ for all experiments.

All tests were done at atmospheric pressure immersed in an air flow of $1,740 \mathrm{~m}^{3} / \mathrm{h}$ forced into the climatic chamber at the selected temperature. The finned profile of the outer shell of the prototype helps the transmission of heat from the device into the air.

Figure 11 shows the $Z$-transmitter prototype assembled and ready for tests according to the first setup.

\section{Test Results}

5.1. Fast Stage Blocked-Maximum Force, Stiffness, and Experimental Force Ratio. In the first setup, both stator and fast stages were tightly fixed to the structure. When the slow stage is moved the $Z$-transmitter tries to move the fast stage exerting a force that is measured by the load cell to which it is attached. Figure 12 shows the force exerted on both slow and fast stages versus slow stage position when the fast stage is blocked at a temperature of $15^{\circ} \mathrm{C}$. The applied input motion was sinusoidal with $9 \mathrm{~mm}$ amplitude and $0.1 \mathrm{~Hz}$ frequency. Once the maximum value of the force is surpassed then the slow stage slides up to its next stable position around which the force-displacement profile is repeated again. This is quite similar to the behavior of rotational magnetic gears when the fast stage is blocked [15]. The significant jump and hysteresis that appears when the sense of motion is reversed can be attributed to the stiffness of the blocking tool and hysteresis of the friction bearings.

Stiffness as defined in (1) is clearly oscillatory with respect to the displacement as it is shown in Figure 13. It becomes zero at the "sliding" positions and maximum at the equilibrium point. Table 1 shows the slow equivalent stiffness at equilibrium of both slow and fast stages and the experimental force ratio at equilibrium as well.

This kind of test was repeated up to $91^{\circ} \mathrm{C}$. Both maximum force and experimental force ratio are shown in Figure 14. Clearly, the experimental force ratio remains constant with an 


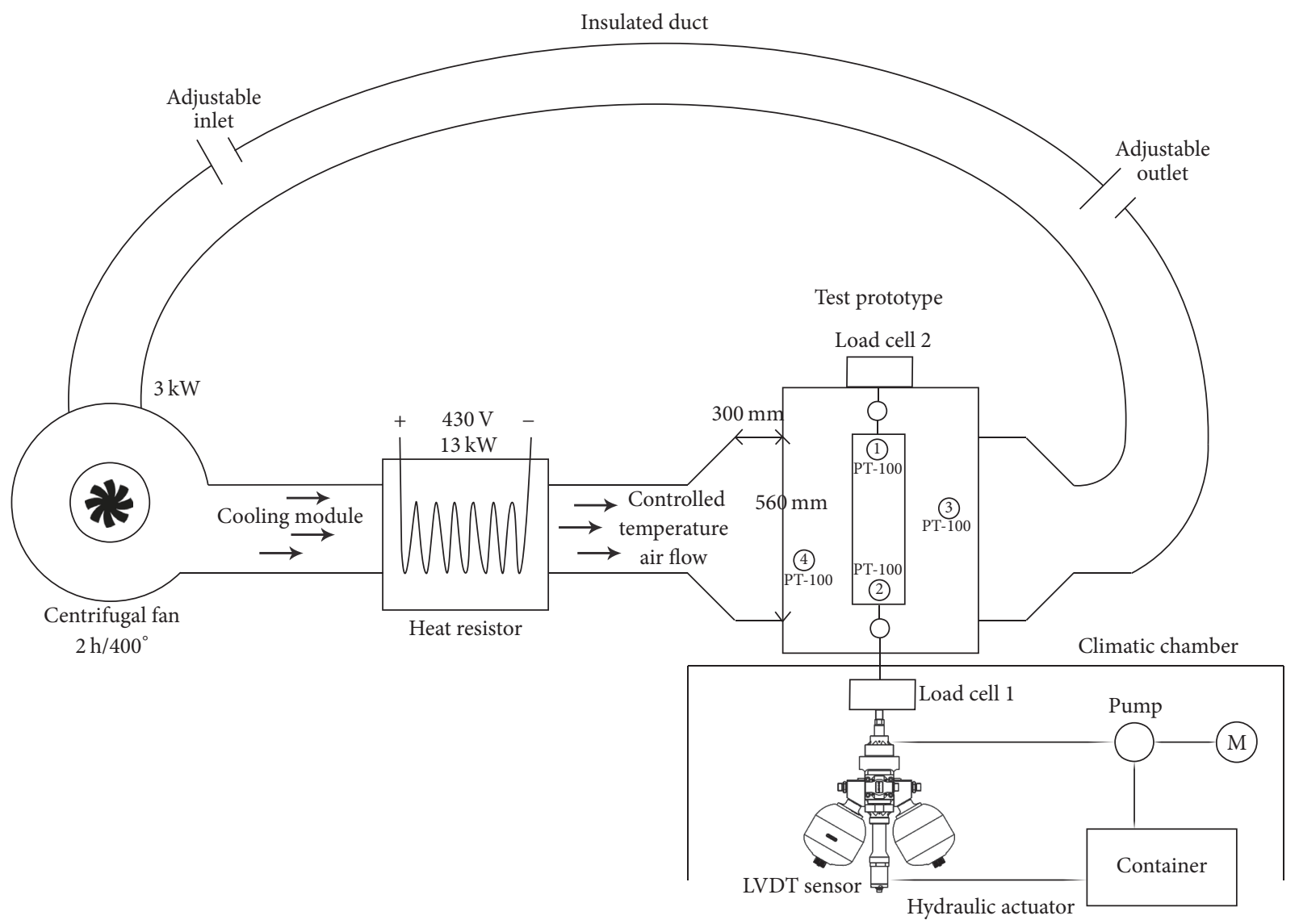

FIGURE 7: Main test bench components scheme.

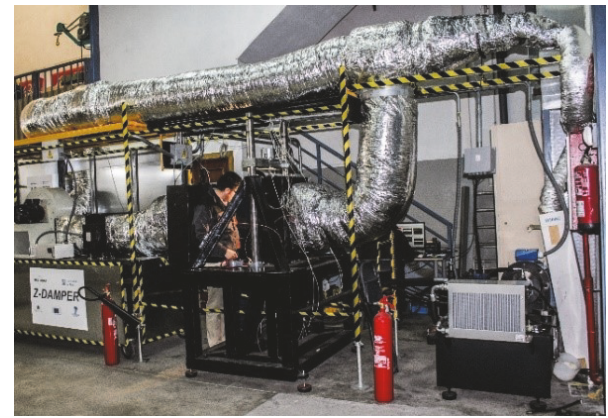

Figure 8: Operating test bench.

average value of $7.0 \pm 0.3$ while the maximum force decreases by $2.9 \pm 0.2 \mathrm{~N} /{ }^{\circ} \mathrm{C}$. This phenomenon is due to the dependence of magnetization on temperature. Magnetization decreases with temperature and, therefore, magnetic forces decrease as well with temperature.

5.2. Free Fast Stage. In the second setup, the fast stage can move freely while the slow stage is driven in a sinusoidal way by the hydraulic actuator. Frequency was swept from 0.1 up to $50 \mathrm{~Hz}$. Figure 15 shows the position of both slow and fast stages in a sequence of increasing frequency and Figure 16 shows the amplitude of the fast stage movement relative to seven times the amplitude of the slow stage versus frequency. The amplitude of the movement of the slow stage in Figure 15 is $\pm 4.7 \mathrm{~mm}$ and it can be clearly seen that the movement of the fast stage is multiplied by seven. The amplitude of the slow stage in Figure 16 is kept much smaller throughout the test $( \pm 0.7 \mathrm{~mm})$ to prevent the fast stage from surpassing the maximum stroke at the resonance.

The normalized displacement of the fast stage fits that of a damped harmonic oscillator with natural frequency $\omega=18 \mathrm{~Hz}$ and damping coefficient of $\xi=0.17$. This is in good agreement with the theoretical value expected if we assume it is a harmonic oscillator with a stiffness equal to that previously measured for the first setup $\left(K_{F}=48 \pm 8 \mathrm{~N} / \mathrm{mm}\right)$ and a mass of the fast stage $m_{F}=3.10 \pm 0.05 \mathrm{~kg}$ that provides $\omega_{0}=\sqrt{K_{F} / m_{F}}=19 \pm 2 \mathrm{~Hz}$.

The resonant behavior is therefore conditioned by the fast stage mass and stiffness. It is clearly detected as a resonant peak for the force exerted on the slow stage as can be shown in Figure 17. In this case, the amplitude of the oscillation at the slow stage was limited to $0.7 \mathrm{~mm}$ to prevent surpassing the fast stage limit at the resonance condition.

If the amplitude were larger, the fast stage would reach the maximum limit and surpass it; nothing breaks but the $Z$ transmitter is not able to exert a force higher than its limit. There will be a saturation of the device. For instance, Figure 18 


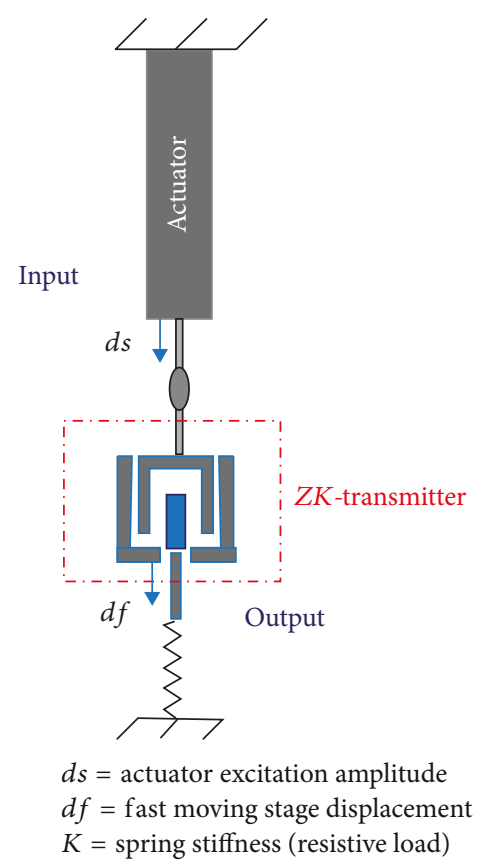

FIgURE 9: Schematic representation.

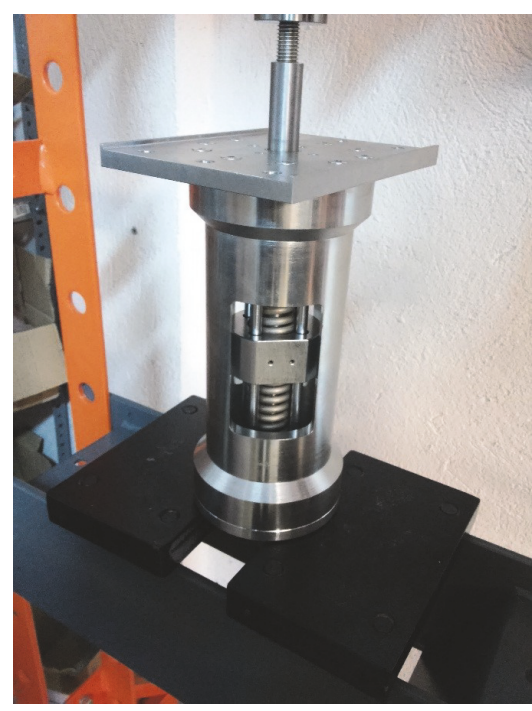

FIGURE 10: Spring used for the setup.

shows the case for a $1 \mathrm{~mm}$ amplitude for the slow stage. There is a clear saturation of the force on the slow stage without a clear resonant peak as in the previous case.

5.3. Third Setup: General Impedance Matching. Mechanical impedance is defined as the ratio between the amplitudes of force and speed in a vibration movement. The two previous setups were limit cases of infinite and zero mechanical impedance attached to the fast stage. The effectiveness of the $Z$-transmitter matching real mechanical impedances was eventually measured using this third setup. The objective was to characterize the input force to be applied to slow stage

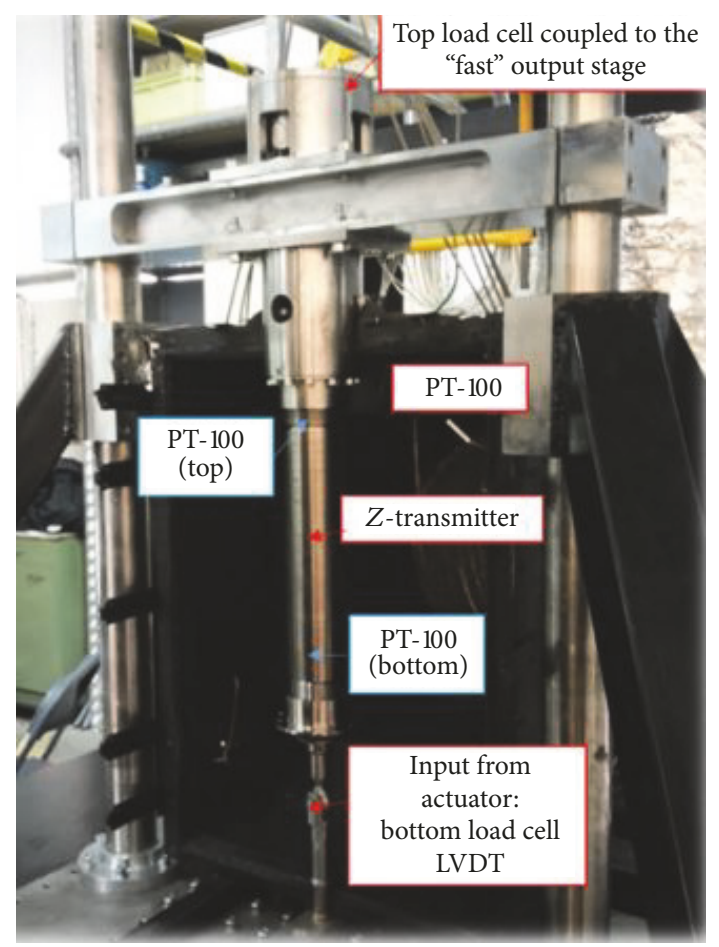

FIGURE 11: $Z$-transmitter first setup.

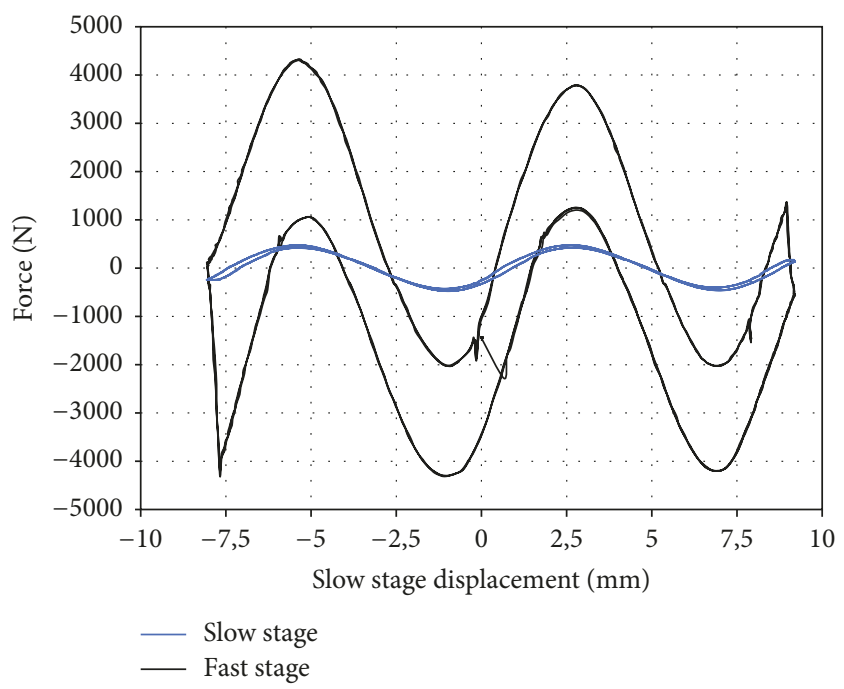

FIGURE 12: Slow and fast force versus slow stage displacement during quasistatic $(0.1 \mathrm{~Hz})$ tests at room temperature.

to move it at a defined frequency and amplitude when the fast stage of the $Z$-transmitter is coupled to a set of three mechanical springs with different stiffness $K_{C}(10,50$ and $100 \mathrm{~N} / \mathrm{mm}$ ) in a dynamic range from $0.1 \mathrm{up}$ to $50 \mathrm{~Hz}$. The resonance frequency is expected to be like that of a harmonic oscillator with a stiffness sum of that of coupled spring $K_{C}$ and that of the fast stage $K_{F}$ and a mass sum of those of the fast stage and the mechanical attachment to the spring. 


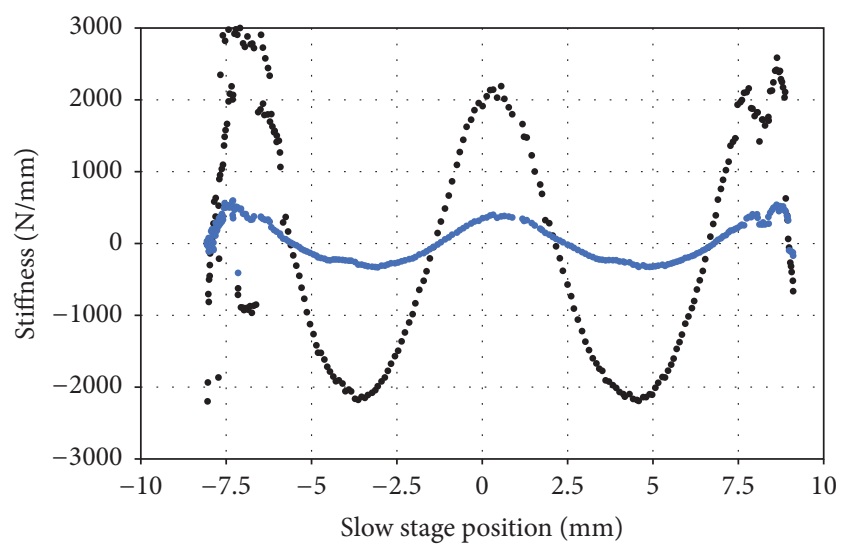

- Slow stage stiffness

- n. Fast stage stiffness

FIgURE 13: Slow and fast stages stiffness versus slow stage displacement during quasistatic $(0.1 \mathrm{~Hz})$ tests at room temperature.

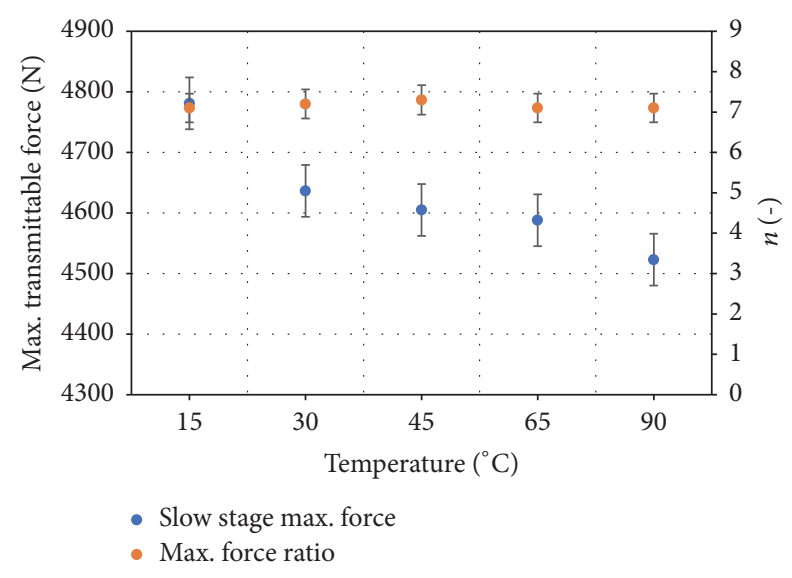

FIGURE 14: Maximum slow stage force versus experimental force.

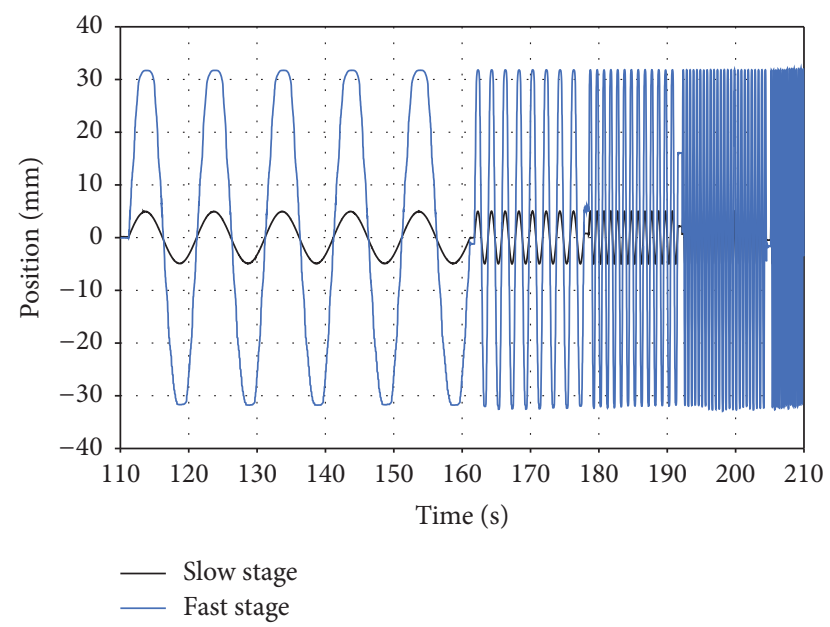

FIGURE 15: Slow stage and fast stage position versus time during $Z$ transmitter dynamic tests.

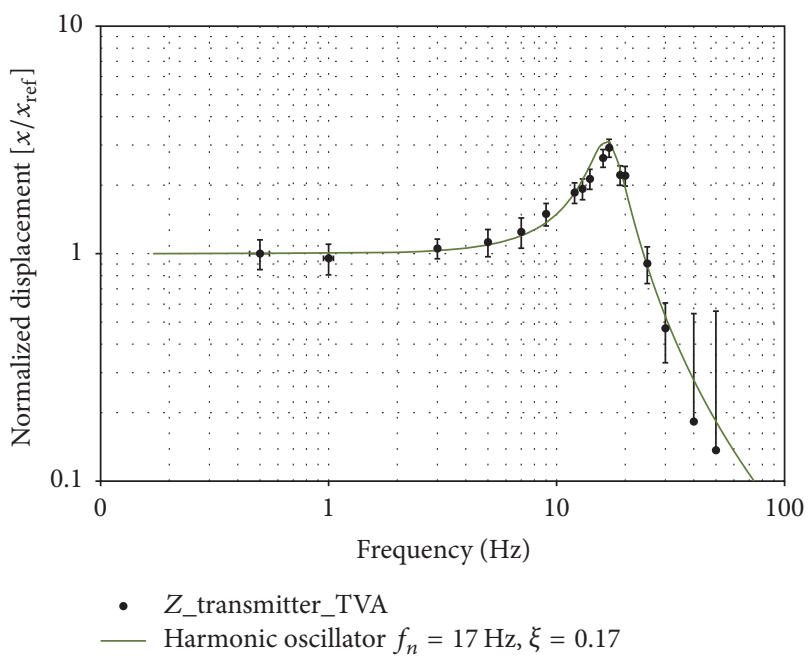

FIGURE 16: Normalized displacement versus frequency during $Z$ transmitter tests.

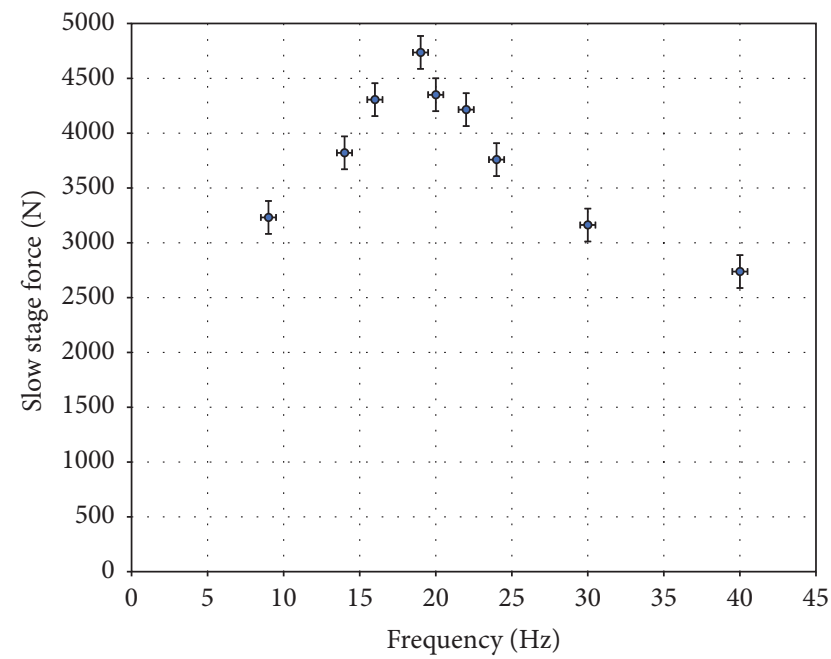

Figure 17: Slow stage force versus frequency for the $Z$-transmitter with $0.7 \mathrm{~mm}$ input amplitude.

The experimental resonant frequency is in good agreement with that calculated in such a way as summarized in Table 2.

The experimental values for the resonant frequency are easily found measuring the amplitude of displacement of the fast stage while keeping the amplitude of the slow stage constant. Figure 19 shows the normalized displacement of the fast stage versus frequency for these three cases when the amplitude of the displacement of the slow stage is $2.5 \mathrm{~mm}$. The experimental resonant peaks are in good agreement with those predicted.

The linearity of the force/speed ratio for both slow and fast stages was tested as well.

Figure 20 shows both force on the slow stage and force on the fast stage versus amplitude of their speed at $1 \mathrm{~Hz}$ for different displacement amplitudes when attached to the 
TABLE 2: Summary of coupled stiffness.

\begin{tabular}{lccc}
\hline $\begin{array}{l}\text { Coupled stiffness } \\
K_{C}[\mathrm{~N} / \mathrm{mm}]\end{array}$ & $\begin{array}{c}\text { Total stiffness } \\
\text { (magnetic }+ \\
\text { coupled) }[\mathrm{N} / \mathrm{mm}]\end{array}$ & $\begin{array}{c}\text { Expected resonant } \\
\text { frequency }[\mathrm{Hz}]\end{array}$ & $\begin{array}{c}\text { Experimental } \\
\text { resonant freq. }[\mathrm{Hz}]\end{array}$ \\
\hline $10.1 \pm 0.2$ & $58 \pm 8$ & $17 \pm 2$ & $16 \pm 2$ \\
$50.0 \pm 2$ & $98 \pm 10$ & $22 \pm 2$ & $19 \pm 2$ \\
$100.0 \pm 2$ & $148 \pm 10$ & $27 \pm 2$ & $27 \pm 2$ \\
\hline
\end{tabular}

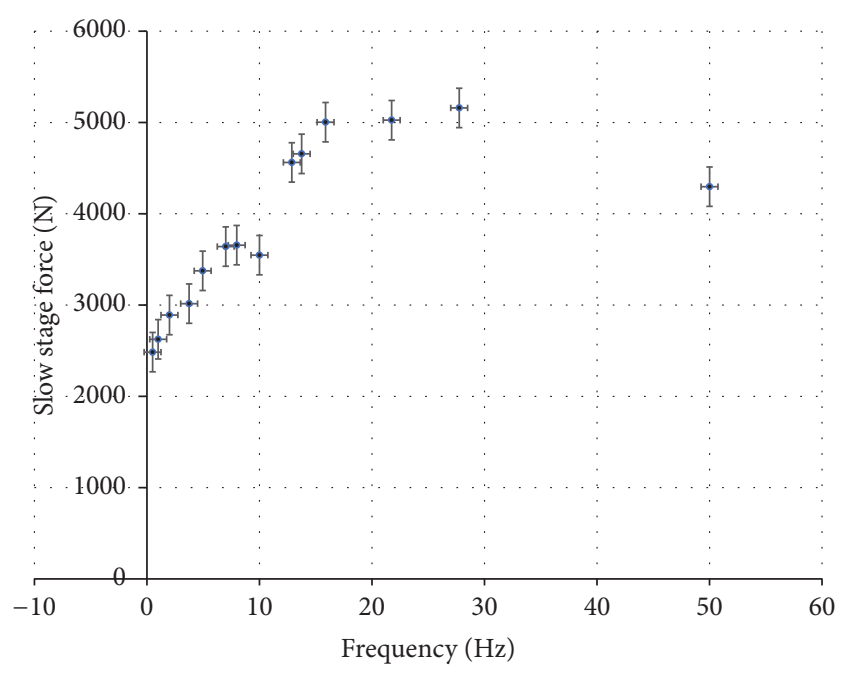

FIGURE 18: Slow stage force versus frequency for the $Z$-transmitter with $0.7 \mathrm{~mm}$ input amplitude.

$50 \mathrm{kN} / \mathrm{m}$ spring. The four points plotted for the slow stage correspond to those four points plotted for the fast stage.

For a perfect impedance transformer, the force exerted on the slow stage should be 7 times larger than the force exerted by the fast stage and the speed should be 7 times lower than the fast stage; that is, the input impedance of the slow stage should be $7^{2}=49$ times greater than that of the fast stage. However, it is clear from Figure 17 that the slope of the slow stage force versus speed amplitude for this case of $50 \mathrm{kN} / \mathrm{m}$ and $1 \mathrm{~Hz}$ is only 15.3 times that of the fast stage, instead of the expected 49 times. It is important to remember that the fast stage impedance is that resulting from the combination of the fast stage's own impedance and the spring and mass attached to it.

A possible cause of the nonideal behavior of the $Z$ transmitter is the friction force of the bearings that makes the extrapolated quasistatic slow stage force $(0 \mathrm{~Hz})$ not to be zero as it would be in an ideal system. However, even an impedances ratio of 15.3 makes the device useful enough. It makes sense to use a damper or an active actuator attached to the fast stage to provide a large force effect controlling a small displacement.

\section{Conclusions}

A mechanical impedance matching device based on a linear magnetic gear, so called $Z$-transmitter, has been developed

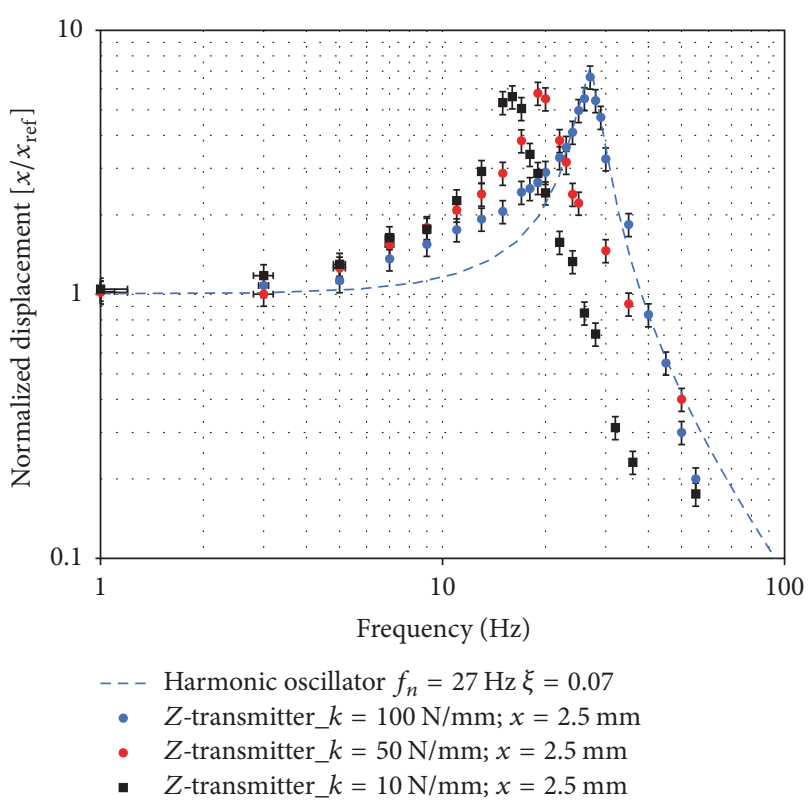

FIGURE 19: Normalized displacement versus frequency for three different motion amplitudes.

and tested experimentally, demonstrating a maximum force capacity of $4700 \mathrm{~N}$ at $25^{\circ} \mathrm{C}$. Maximum force decreases with temperature due to magnetization decrease but force ratio between slow and fast stages remains constant and equal to the design value: 7.0.

The $Z$-transmitter behaves as a mechanical impedance matching device when any stiffness is attached to the fast stage, including the limit cases of a blocked fast stage or a free to move fast stage.

There are intrinsic stiffness and inertia for the fast stage that conditions the mechanical behavior and a finite minimum impedance corresponding to the free case.

The frequency of resonance can be easily estimated for the general case using the data obtained for the blocked fast stage case and the known data of the attached impedance.

However, the real effective impedances ratio values are lower than the theoretical value of 49 . This can be attributed to the friction created by the friction linear bearings provided in the prototype.

The $Z$-transmitter has proved to be a potentially extremely useful tool for vibration control when combined with other elements such as dampers, springs, and/or active elements. Moreover, the existence of a maximum of the transmitted force makes it ideal for shock damping. 


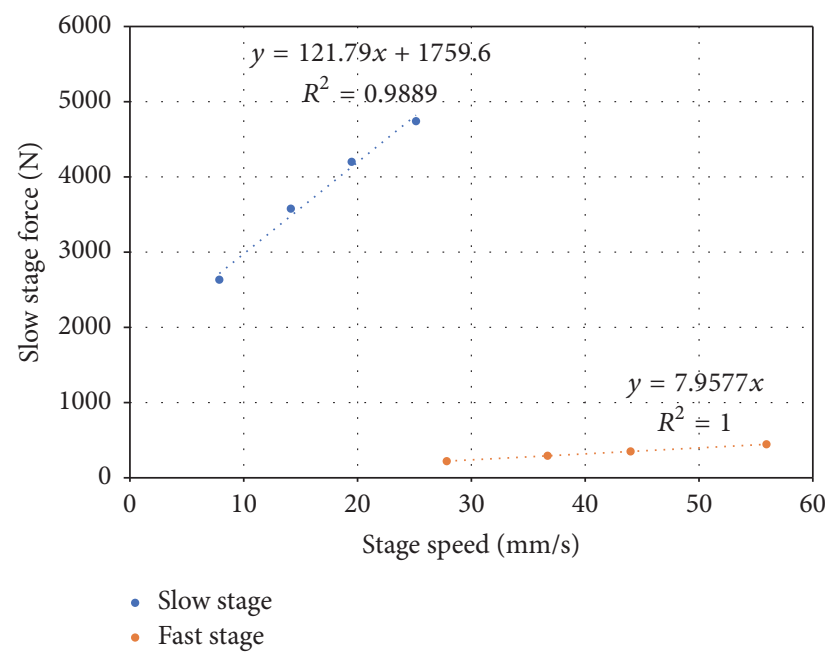

FIGURE 20: Force on slow and fast stages versus amplitude of speed at $1 \mathrm{~Hz}$ and different displacement amplitudes when the fast stage of the $Z$-transmitter is attached to a $50 \mathrm{kN} / \mathrm{m}$ spring.

\section{Conflicts of Interest}

The authors declare that they have no conflicts of interest.

\section{Acknowledgments}

This work has been funded by the Seventh Framework Clean Sky Program, under Grant JTI-CS-2013-02-SFWA-03-013.

\section{References}

[1] C. Liu, X. Jing, S. Daley, and F. Li, "Recent advances in microvibration isolation," Mechanical Systems and Signal Processing, vol. 56, no. 1, pp. 55-80, 2015.

[2] E. I. Rivin, Passive Vibration Isolation, ASME Press, 2003.

[3] N. Orǎşanu, A. Craifaleanu, and C. Dragomirescu, "Theoretical and experimental studies on magnetic dampers," Applied Mechanics and Materials, vol. 430, pp. 351-355, 2013.

[4] A. Bissal, E. Salinas, J. Magnusson, and G. Engdahl, "On the Design of a Linear Composite Magnetic Damper," IEEE Transactions on Magnetics, vol. 51, no. 11, 2015.

[5] B. Ebrahimi, M. B. Khamesee, and M. F. Golnaraghi, "Design and modeling of a magnetic shock absorber based on eddy current damping effect," Journal of Sound and Vibration, vol. 315, no. $4-5$, pp. 875-889, 2008.

[6] C. B. Churchill, D. W. Shahan, S. P. Smith, A. C. Keefe, and G. P. McKnight, "Dynamically variable negative stiffness structures," Science Advances, vol. 2, no. 2, Article ID e1500778, 2016.

[7] R. A. Ibrahim, "Recent advances in nonlinear passive vibration isolators," Journal of Sound and Vibration, vol. 314, no. 3-5, pp. 371-452, 2008.

[8] C. Yilmaz and N. Kikuchi, "Analysis and design of passive bandstop filter-type vibration isolators for low-frequency applications," Journal of Sound and Vibration, vol. 291, no. 3-5, pp. 1004-1028, 2006.

[9] J. L. Pérez-Díaz et al., "Enhanced magnetic vibration damper with mechanical impedance matching," 2015.
[10] J. L. Pérez-díaz, I. Valiente-blanco, and C. Cristache, "ZDamper: a new paradigm for attenuation of vibrations," 2016.

[11] S. Miladinović et al., "The development of magnetic gears for transportation applications," Mobility and Vehicle Mechanics, 2017.

[12] J.-L. Pérez-díaz, J. C. Garcia-Prada, I. Valiente-Blanco, and E. Diez-Jimenez, "Magnetic-superconductor cryogenic noncontact harmonic drive: Performance and dynamical behavior," in Proceedings of the 4th European Conference on Mechanism Science (EUCOMES '12), pp. 357-364, 2012.

[13] N. I. Berg, "Linear magnetic actuator for vehicle suspension," 2015.

[14] J. Perez-Diaz, E. Diez-Jimenez, I. Valiente-Blanco, C. Cristache, M. Alvarez-Valenzuela, and J. Sanchez-Garcia-Casarrubios, "Contactless Mechanical Components: Gears, Torque Limiters and Bearings," Machines, vol. 2, no. 4, pp. 312-324, 2014.

[15] J. L. Perez-Diaz, E. Diez-Jimenez, C. Cristache et al., "Magnetic non-contact harmonic drive," in Proceedings of the International Mechanical Engineering Congress and Exposition (IMECE '13), 2013. 


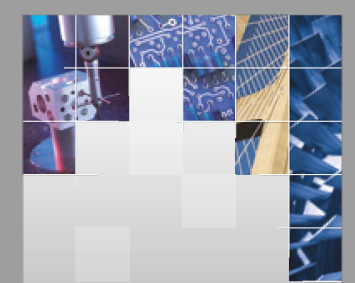

\section{Enfincering}
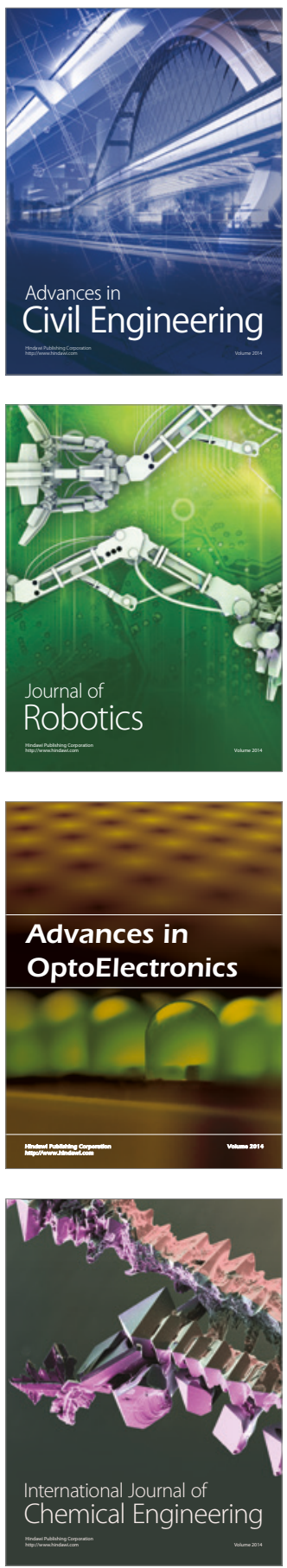

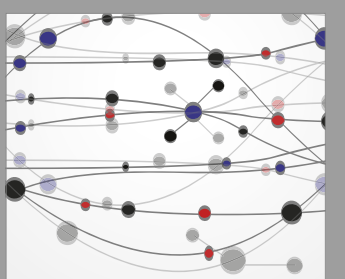

The Scientific World Journal

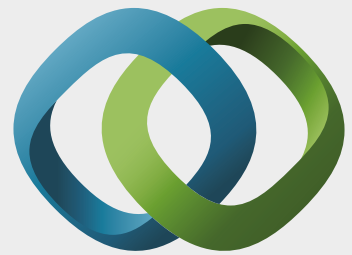

\section{Hindawi}

Submit your manuscripts at

https://www.hindawi.com
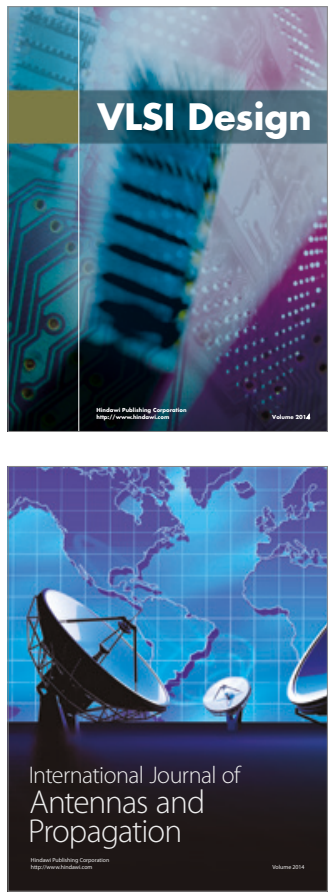

\section{Rotating}

Machinery
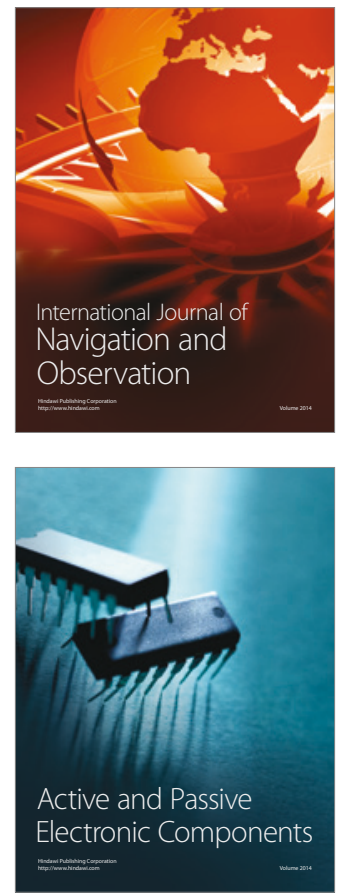
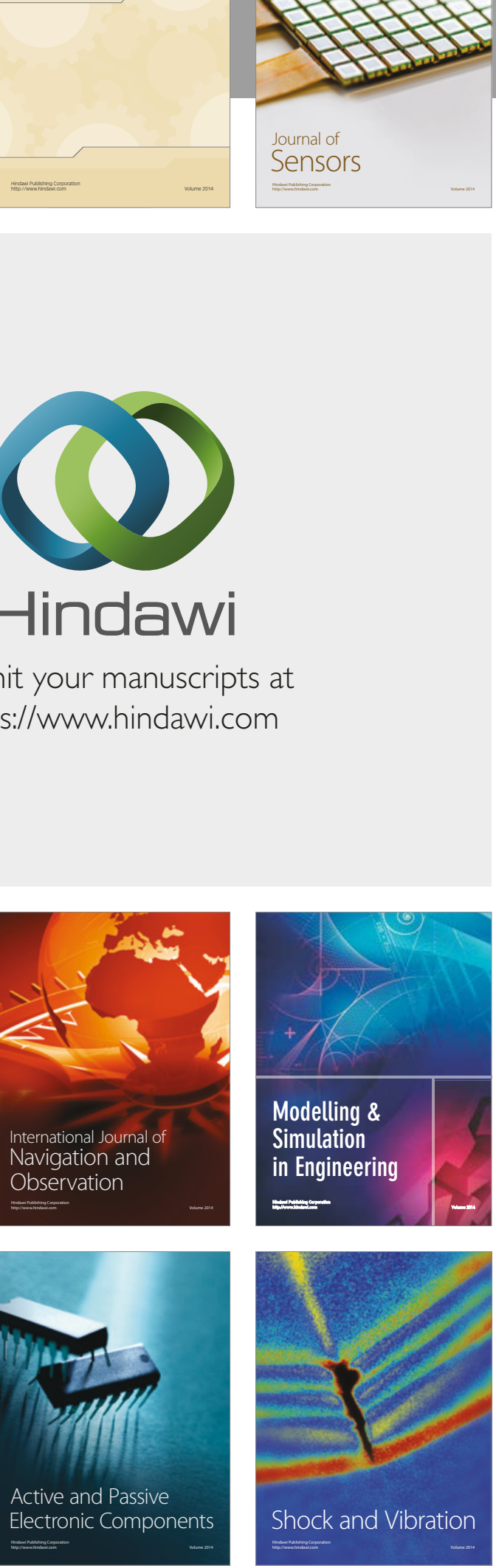
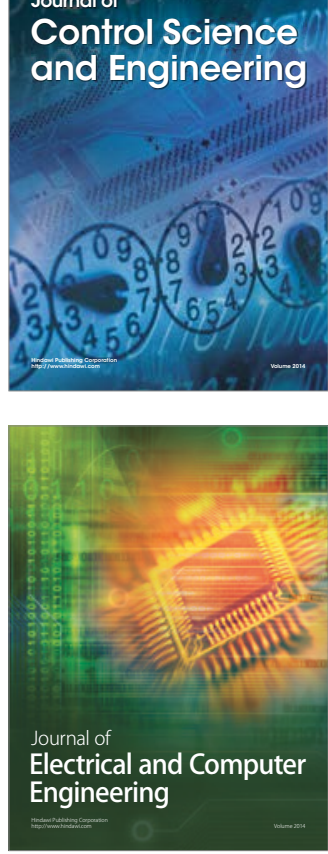

Distributed

Journal of

Control Science

and Engineering
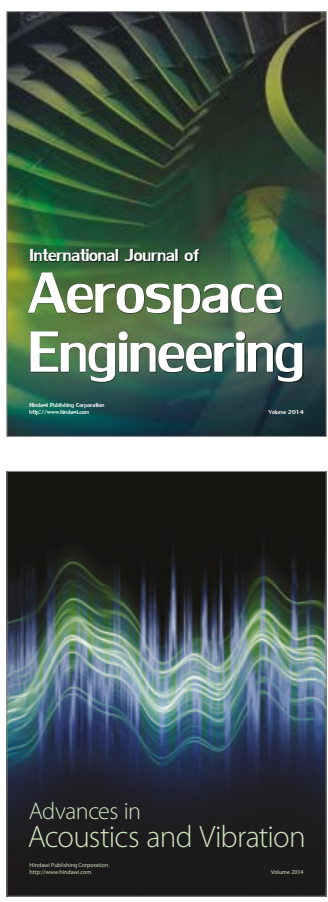

Sensor Networks 